\title{
miR-940 upregulation contributes to human cervical cancer progression through p27 and PTEN inhibition
}

\author{
KE SU, CHUN-FANG WANG, YING ZHANG, YU-JIE CAI, YAN-YAN ZHANG and QIAN ZHAO \\ Department of Gynecology, The First Affiliated Hospital of Zhengzhou University, Zhengzhou, Henan 450052, P.R. China
}

Received November 10, 2016; Accepted January 16, 2017

DOI: $10.3892 /$ ijo.2017.3897

\begin{abstract}
Cervical cancer is considered as a gynecological malignancy accompanied with high rates of mortality across the world. Effective diagnostic, prognostic markers as well as therapeutic targets are necessary to be explored. The p27 and PTEN are known to modulate tumor cell growth and proliferation. However, the molecular mechanisms modulating these genes have not completely been elucidated. In our study, real-time (RT) quantitative PCR indicated that miR-940 levels were upregulated in human cervical cancer tissue samples and cell lines. Overexpression of miR-940 could reduce overall survival in patients. Ectopic miR-940 accelerated cervical cancer cell growth, proliferation and cell cycle arrest in vitro as well as tumor formation in vivo. p27 and PTEN were evidenced as direct targets for miR-940 and inhibition of p27 and PTEN recovered the suppressive function of miR-940silenced cell towards to proliferation and tumorigenicity in cervical cancer cells. In addition, miR-940 expression was inversely associated with p27 and PTEN expression levels and actively with cyclin D1 in cervical cancer specimens. The results from our study demonstrated that miR-940 regulated p27 and PTEN post-transcriptionally and might play a significant role in cervical cancer development and progression. Thus, miR-940 might provide a potential value as therapeutic target for cervical cancer treatment in future.
\end{abstract}

\section{Introduction}

Cervical cancer is known as the third most common malignancy among the female, and about half million new cases are diagnosed and $>200,000$ deaths are reported each year $(1,2)$. Although a large number of cervical cancer cases can be reduced by traditional screening and precancerous lesions treatment, cervical cancer is still the main reason contributing to cancer mortality among females in many developing

Correspondence to: Dr Qian Zhao, Department of Gynecology, The First Affiliated Hospital of Zhengzhou University, 1 Jianshe Road, Zhengzhou, Henan 450052, P.R. China

E-mail: suke450052@qq.com; su@126.com

Key words: cervical cancer, p27 and PTEN, miR-940 regions and countries (3). The high number of cervical cancer death is due to the low survival rates of patients suffered from advanced cervical cancer during diagnosis (4). The strategy for cervical cancer is currently, stage-specific. Although early stage disease could be cured with surgery or radiotherapy, the most useful strategy for the local advanced-stage patients is with co-treatment of chemotherapy and pelvic irradiation $(5,6)$. Recently, reports from large scale genomic sequencing of human cervical tumors has illustrated that many targeted treatments may possess a better choice (7). Therefore, despite the progress made in prevention, patients with cancers in metastasis and those who are experiencing recurrent or persistent disease are without effective therapeutic options (8).

MicroRNAs (miRNAs) are known as endogenous, small 18-25 ng nucleotides. They are non-coding RNAs, negatively modulating gene expression at the level of post-transcription through binding with 3' untranslated region (UTR) involved in specific messenger RNAs (mRNAs) (9-11). Accompanied with sequence complementary to their target mRNAs partially, miRNAs are of great importance in regulating (mostly inhibiting) gene expression in different kinds of organisms $(12,13)$. Additionally, studies have evidenced that miRNAs are included in a variety of biological processes, such as cell growth, proliferation as well as metabolism, which is dependent on mRNA translation inhibition (14). In addition, studies indicate that $\sim 20-30 \%$ human genes are modulated by miRNAs, altering expression of various genes (15). Studies before have revealed the effects of miR-940 on different diseases. Liang et al demonstrated that miR-940 downregulation leads to human Tetralogy of Fallot development (16). Moreover, miR-940 was shown to suppress progression of prostate cancer and pancreatic ductal adenocarcinoma by suppressing migration and invasion enhancer 1 (17). Furthermore, previous report revealed that miR-940 suppressed the migratory and invasive activity of cells, attenuated anchorage-independent growth ability, accompanied with increased E-cadherin activation in prostate cancer of human (18). Taken together, by targeting different genes under different pathological conditions, miR-940 might exhibit different biological functions and clinical impacts. Hence, we should explore the role of miR-940 in a disease-specific manner.

Herein, we elucidated that miR-940 is markedly increased in cervical cancer and that miR-940 expression is related to the development and overall survival rate in cervical cancer. Ectopic miR-940 promoted, while inhibition of miR-940 
reduced the proliferation, cell cycle arrest and tumorigenicity of cervical cancer cells from human in vitro. Furthermore, we demonstrated that miR-940 directly targets and downregulates p27 and PTEN through recognizing their 3' UTRs, and downregulation of p27 and PTEN was essential for the miR-940-mediated effects in cervical cancer cells. This study demonstrated that miR-940 shows an essential role in human cervical cancer development and may be a potential target for the treatment of cervical cancer.

\section{Materials and methods}

Cancer cell culture. Human cervical cancer cell lines, including $\mathrm{SiHa}, \mathrm{HeLa}, \mathrm{Caski,} \mathrm{C} 4-1$ and C-33a, and the normal cervical cells of CEC were purchased from American Type Culture Collection, the Cell Resource Center, Shanghai Institute of Biochemistry and Cell Bank at the Chinese Academy of Sciences. Cell lines were routinely authenticated by DNA-fingerprinting and isoenzyme analyses and checked for contamination by mycoplasma using Hoechst staining. All cell lines were maintained in Roswell Park Memorial Institute (RPMI)-1640, Dulbecco's modified Eagle's medium or minimum essential medium with $10 \%$ fetal bovine serum (FBS) and then were cultured at $37^{\circ} \mathrm{C}$ with $5 \% \mathrm{CO}_{2}$.

Tissue specimens and patient information. The paraffinembedded and -archived cervical cancer specimens and freshly collected cervical cancer tissue specimens evaluated in this study were histopathologically and clinically diagnosed at the Xinjiang Medical University Affiliated Tumor Hospital between 2010 and 2014. The disease stages of all patients were divided following the International Federation of Gynecology and Obstetrics (FIGO) guidelines. The clinical characteristics of these 83 patients are provided in Table I. Normal cervical tissues were obtained from individuals who underwent wedge biopsy of the cervix and proved to be free of any pre-existing detectable situations pathologically. All samples were detected along with prior written informed consent from the patients. Prior donor consents, and approval from Institutional Research Ethics Committee were received.

RNA extraction and real-time quantitative PCR. Total RNA from tumor tissue samples and cultured cells was extracted through mirVana miRNA Isolation kit (Ambion) according to the manufacturer's instructions. Then cDNA was synthesized from total RNA through TaqMan miRNA reverse transcription kit obtained from Applied Biosystems (USA). RT PCR was performed by the Applied Biosystems 7500 Sequence Detection system with iQ ${ }^{\mathrm{TM}}$ SYBR Green Supermix (Bio-Rad Laboratories, USA) with $5 \mathrm{ng}$ cDNA as well as $10 \mathrm{pM}$ of each primer for assessment. The data here were then normalized to geometric mean of the housekeeping gene of GAPDH or U6 small nuclear RNA expression and the results were evaluated with the $2^{-\triangle \Delta C T}$ method. Sequences of the primers used are as follows: miR-940 forward, 5'-GCA TCG TTC CTT CAA GCC GAT CT-3' and reverse, 5'-TGG GTG AGT CGT TCG G-3'; U6 forward, 5'-GTC CTG GCA GAT ATA CAC TAA ACA T-3' and reverse, 5'-CTC ACG CTT GAA TTC ATG CGG CTT-3'; PTEN forward, 5'-TGT TTG GCA GAT CTT CCT TG-3' and reverse, 5'-CTC GGT CGT CGC TCA TAT-3'; p27 forward, 5'-TTG TTG AGC GTA TCT CTT CG-3' and reverse, 5'-CGT CGT CAG TCT ATC GCT-3'; cyclin D1 forward, 5'-GTT GGT TCA TAG CTC TGT CAT-3' and reverse, 5'-CTG CGT CGT TAC GTC CTA-3'; Bax forward, 5'-GCT GAT TTA TAG CAC CGT CAT TG-3' and reverse, 5'-CAG CAT GGT TTC GAC CGA-3'; Bcl-xL forward, 5'-CTT GCT TAA TCG CCC TAT CGC AT-3' and reverse, 5'-TTG CAT GGT AAC CTC CTG AAC-3'; GAPDH forward, 5'-CAT TCA AGA CCG GAC AGA GG-3' and reverse, 5'-ACA TAC TCA GCA CCA GCA TCA CC-3'.

Oligonucleotides, siRNA and transfection. In order to produce a miR-940 expression vector, the miR-940 antisense (miRZip-940) plasmid used as miR-940 inhibitor, and the vector control (miRZip-vector) were purchased from System Biosciences (San Francisco, CA, USA) and used according to the manufacturer's instructions. For depletion of p27-, and PTEN-siRNAs were synthesized and purified by RiboBio. Transfection of oligonucleotides and siRNAs were performed using the Lipofectamine 2000 reagent (Invitrogen, USA), according to the manufacturer's instructions. miR-940 mimics, and the negative control were obtained from Genecopoeia (USA) and transfected into cervical cancer cells by the use of Lipofectamine 2000 reagent (Invitrogen), according to the manufacturer's instructions.

Immunoblot analysis. Cell proteins were isolated by a T-PER Tissue Protein Extraction reagent kit (Thermo) according to the manufacturer's instructions. Different protein concentrations were calculated through BCA protein assay kit. Then the equal mounts of protein were loaded per well on a $10-12 \%$ sodium dodecyl sulphatepolyacrylamide gel. Next, proteins were transferred onto the polyvinylidene difluoride membrane (PVDF). Resulting membrane was then blocked by the use of Tris-buffered saline with $0.05 \%$ Tween-20 (TBS-T), dissolved in 5\% skim milk (Sigma, USA) at room temperature for $3 \mathrm{~h}$ on a rotary shaker, which was followed by TBS-T for washing. Specific primary antibody used in our study, suspended in TBST, was then incubated with the membrane at $4^{\circ} \mathrm{C}$ overnight. Next, the membrane was washed by the TBS-T after incubation with peroxidaseconjugated secondary antibody at room temperature for $2 \mathrm{~h}$. The immunoactive proteins were detected through an enhanced chemiluminescence western blotting detection kit. Western blotting bands were calculated through GE Healthcare ECL western blot analysis system and exposed to X-ray film (Kodak). The primary antibodies were: p27 (Abcam), PTEN (Abcam, USA), caspase-3 (Cell Signaling Technology), caspase-9 (Abcam), PARP (Abcam), Bcl-2 (Cell Signaling Technology), Bcl-xL (Cell Signaling Technology), Mcl-1 (Abcam), Bak (Cell Signaling Technology), Bad (Cell Signaling Technology), Bax (Abcam) and cyclin D1 (Cell Signaling Technology, USA).

Colony formation assay. Cervical cancer cells per well in 60-mm plates were cultured in 10\% FBS RPMI-1640. Cells were treated under different conditions. After another 7-day incubation, the cell colonies were washed twice with PBS, fixed with $4 \%$ paraformaldehyde for $15 \mathrm{~min}$ and then stained 
Table I. Clinical parameters of 83 cervical cancer patients.

\begin{tabular}{|c|c|c|}
\hline Category & Subcategory & $\mathrm{N}=83$ \\
\hline \multirow[t]{2}{*}{ Age (years) } & $\leq 45$ & 32 \\
\hline & $>45$ & 51 \\
\hline \multirow[t]{2}{*}{ Tumor size } & $\leq 5 \mathrm{~cm}$ & 58 \\
\hline & $>5 \mathrm{~cm}$ & 26 \\
\hline \multirow[t]{4}{*}{ FIGO stage } & I & 22 \\
\hline & II & 12 \\
\hline & III & 34 \\
\hline & IV & 15 \\
\hline \multirow[t]{3}{*}{ Histology } & Squamous & 75 \\
\hline & Adenocarcinoma & 6 \\
\hline & Adenosquamous & 2 \\
\hline \multirow[t]{3}{*}{ Pelvic node involvement } & Positive & 26 \\
\hline & Equivocal & 14 \\
\hline & Negative & 43 \\
\hline \multirow[t]{2}{*}{ Overall survival } & Death & 24 \\
\hline & Alive & 59 \\
\hline \multicolumn{3}{|l|}{ miR-940 expression } \\
\hline \multirow[t]{4}{*}{ Low expression } & I & 15 \\
\hline & II & 6 \\
\hline & III & 13 \\
\hline & IV & 4 \\
\hline \multirow[t]{4}{*}{ High expression } & $\mathrm{I}$ & 7 \\
\hline & II & 6 \\
\hline & III & 21 \\
\hline & IV & 11 \\
\hline
\end{tabular}

by Giemsa for $30 \mathrm{~min}$. Each clone with $>50$ cells were evaluated. Clone forming efficiency for cells was calculated based on colonies/number of inoculated cells x $100 \%$.

Transwell migration assay. Cervical cancercells $1 \times 10^{5}$ cells/well were seeded in the top chamber of 24-well Transwell micropore polycarbonate membrane filter with pore size of $8-\mu \mathrm{m}$ (Millipore, USA). Then cells were suspended in serum-free medium. Forty-eight hours later, the cells on the top surface of membrane were removed by a cotton swab carefully. Finally, the migrated cells were counted in five random fields for each treatment.

Flow cytometry analysis. All cancer cells in a culture dish were harvested by trypsinization, washed in ice-cold PBS, and fixed in $80 \%$ ice-cold ethanol in PBS. To evaluate the number of cells experiencing apoptosis, the Annexin V-FITC kit (BD Biosciences, USA) was used according to the manufacturer's instructions. Finally, $400 \mu \mathrm{l}$ binding buffer was added to analyze the cells immediately through flow cytometry (BD Biosciences). All experiments were performed in triplicate.
Immunohistochemistry. At the end of our study, the mice were sacrificed. Tumors, kidneys and livers were carefully harvested and maintained in 4\% neutral formalin liquid, IHC staining for the measurement of PTEN, P27 and 1 expression was carried out. The nuclei were counterstained with Mayer's hematoxylin.

Immunofluorescent analysis. Cells were fixed in 4\% paraformaldehyde and then stained overnight with primary antibodies against. Afterwards, cells were washed with phosphate-buffered saline and incubated for $0.5 \mathrm{~h}$ at room temperature with Alexa 488 (Invitrogen) secondary antibodies. Cells were fixed with the ProLong gold antifade reagent with 4',6-diamidino2-phenylindole (Invitrogen) at room temperature for $24 \mathrm{~h}$ before visualizing. The coverslips were viewed through a laser-scanning confocal microscope.

Luciferase assay. Cancer cells were seeded in triplicate in 24 -well plate and allowed to settle for $\sim 12 \mathrm{~h}$. One hundred nanograms of pGL3-PTEN, or p27-luciferase plasmid was cotransfected into cervical cancer cells with TK-Renilla plasmid as control signals using the Lipofectamine 2000 reagent according to the manufacturer's instructions. Luciferase and control signals were measured at $48 \mathrm{~h}$ after transfection using the Dual Luciferase Reporter assay kit (Promega, Madison, WI, USA), according to a protocol provided by the manufacturer. Three independent experiments were performed.

Xenografted tumor model. The mouse experiments were conducted in the Animal Laboratory Center. Female, 4-5-week-old BALB/c-nude mice (18-20 g) were purchased from the Center of Experimental Animal of Xinjiang University. The BALB/c nude mice were randomly divided into two groups. One group of mice was subcutaneously inoculated with $5 \times 10^{6} \mathrm{SiHa} /$ vector cells in the left dorsal flank and with $5 \times 10^{6} \mathrm{SiHa} / \mathrm{miR}-940$ cells in the right dorsal flank of each mouse. Another group was subcutaneously inoculated with $5 \times 10^{6} \mathrm{SiHa} / \mathrm{miRZip}$-vector cells in the left dorsal flank and with $5 \times 10^{6} \mathrm{SiHa} / \mathrm{miRZip}-940$ cells in right dorsal flank of a mouse. Tumor size was calculated with digital caliper. The tumor volume was determined every 5 days and the experimantal mice were sacrificed at the end of 6 weeks. Tumors were then excised, weighed, fixed with $10 \%$ neutral formalin, and then embedded in paraffin for further analysis.

Statistical analysis. The differences of data are stated by means \pm SEM. Different groups were compared through Graph Pad PRISM (Graph Pad Software, USA). P<0.05 was considered as significant difference between groups.

\section{Results}

miR-940 is upregulated and related to overall survival in human cervical cancer. In our study, miR-649, miR-338, miR-21, miR-222, miR-133b, miR-20a, Mir-221, miR-942, miR-353, miR-370, miR-126, and miR-940 were assessed, and miR-940 was upregulated significantly via analyzing a published microarray-based high throughput assessment (NCBI/E-MTAB-1067) $(\mathrm{P}=0.0002)$ in human cervical cancer tissues in comparison to the normal cervical tissues (Fig. 1A). 
A

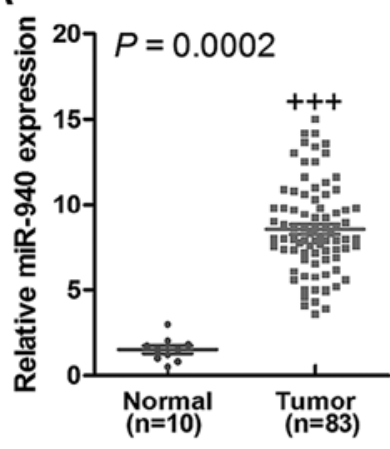

B

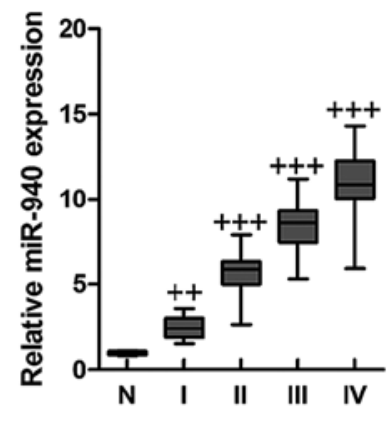

C

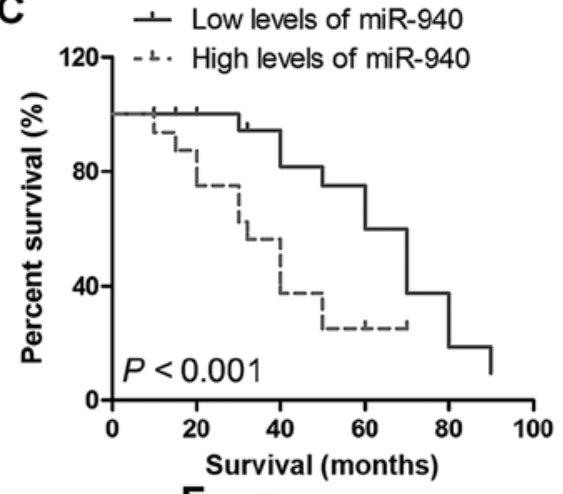

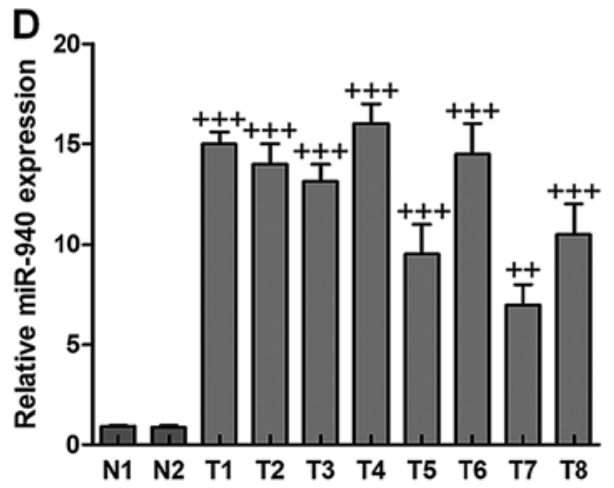
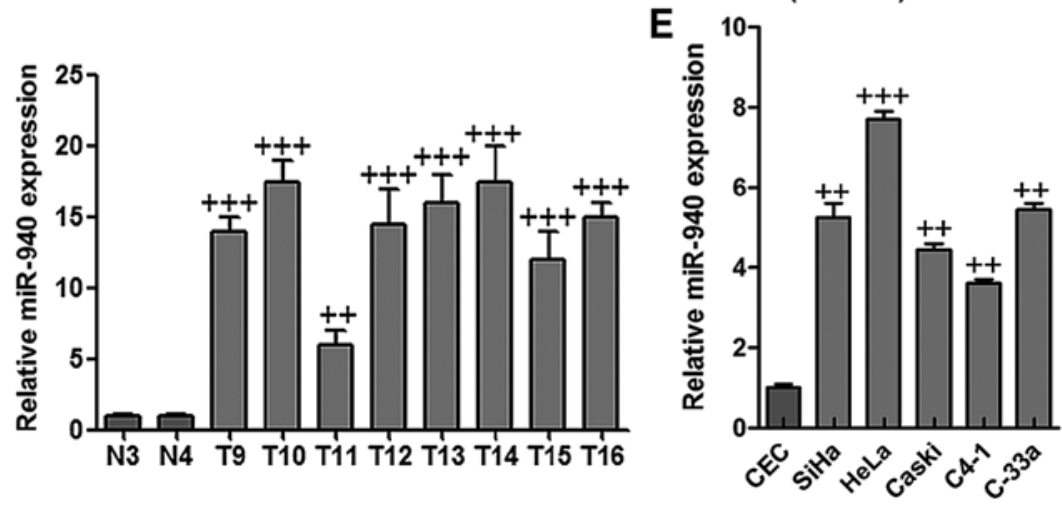

Figure 1. miR-940 is increased in cervical cancer. (A) miRNA-940 was increased in human cervical cancer tissues (n=83) compared with normal cervical tissues ( $\mathrm{n}=10$, and $\mathrm{P}<0.001)$. (B) Real-time PCR analysis of miR-940 expression levels in different clinical stages of human cervical cancer. Transcript levels were normalized to the control of U6 expression. The boundaries of the boxes represent the lower and upper quartiles; the lines within the boxes and whiskers demonstrate the median and outer limits, respectively $(n \geq 10)$. (C) Analysis of overall survival for patients with cervical cancer stratified by low miR-940 and high miR-940 expression. (D) Real-time PCR analysis of miR-940 expression in 8 freshly isolated cervical cancer tissue specimens and two normal cervical tissue specimens. (E) Real-time PCR analysis of miR-940 expression in cervical cancer cell lines and a normal cervical cell line. Transcript levels were normalized to loading control U6 expression. Data are expressed as the mean \pm SEM. ${ }^{+} \mathrm{P}<0.05,{ }^{++} \mathrm{P}<0.01,{ }^{+++} \mathrm{P}<0.001$ versus the normal control.

Expression of miR-940 was further examined in archived clinical cervical cancer specimens. As shown in Fig. 1B, miR-940 expression was low in stage I and II tumors, markedly increased in stage III tumors and was further elevated in stage IV cervical cancer $(n=10)$. In addition, a high level of miR-940 expression was significantly related to shorter overall survival $(\mathrm{P}<0.001)$ (Fig. 1C). The data indicated a possible link between high-level miR-940 expression and the progression of human cervical cancer, and highlights miR-940 may have potential value as a prognostic biomarker in human cervical cancer. Real-time PCR analysis revealed that miR-940 was significantly overexpressed in 16 freshly-collected cervical cancer samples compared to the two normal cervical tissues (Fig. 1D). In line with these observations, upregulation of miR-940 was confirmed in cervical cancer cell lines compared with a control normal cervical cell line (Fig. 1E). Taken together, the results above strongly indicated that miR-940 was upregulated in human cervical cancer.

Overexpression of miR-940 promotes the proliferation and cell cycle progression in human cervical cancer cells. The data indicated that both in $\mathrm{SiHa}$ and HeLa cells, miR-940 was highly expressed compared to the vector control, indicating the stable transfectant expresses miR-940. Furthermore, miR-940 was also reduced by knockdown with significant difference compared to the vector control group (Fig. 2A). The MTT assay demonstrated that ectopic overexpression of miR-940 significantly increased the growth rate of both $\mathrm{SiHa}$ and HeLa cells. Also, miR-940 at low expression dispalyed lower $\mathrm{SiHa}$ and HeLa cell viability compared to the control ones with significant difference (Fig. 2B). The colony formation assay revealed that ectopic overexpression of miR-940 markedly enhanced the growth ability of both SiHa and HeLa cells, as indicated by increased colony numbers and sizes. Similarly, after miR-940 suppression, the percent of colony formation was significantly reduced, which was comparable to the control group in both $\mathrm{SiHa}$ and HeLa cancer cells (Fig. 2C). Furthermore, cell cycle analysis showed ectopic overexpression of miR-940 significantly increased the percentage of cells in the $\mathrm{S}$ phase and decreased the percentage of cells in the G1/G0 peak (Fig. 2D). Collectively, these results demonstrated that miR-940 functioned to promote proliferation and cell cycle progression in human cervical cancer cells.

miR-940 enhances tumorigenicity of cervical cancer cells both in vitro and in vivo. Next, we examined whether miR-940 has any effect on cervical cancer cell migration and invasion. Cervical cancer cells stably expressing miR-940 formed higher numbers and greater migration and invasion than the control cells, while inhibition of miR-940 led to the formation of fewer and less migration and invasion (Fig. 3A and B). The biological effect of miR-940 on cervical cancer progression was further examined using an in vivo tumor model. The miR-940-transduced cervical cancer cells and miR-940- 

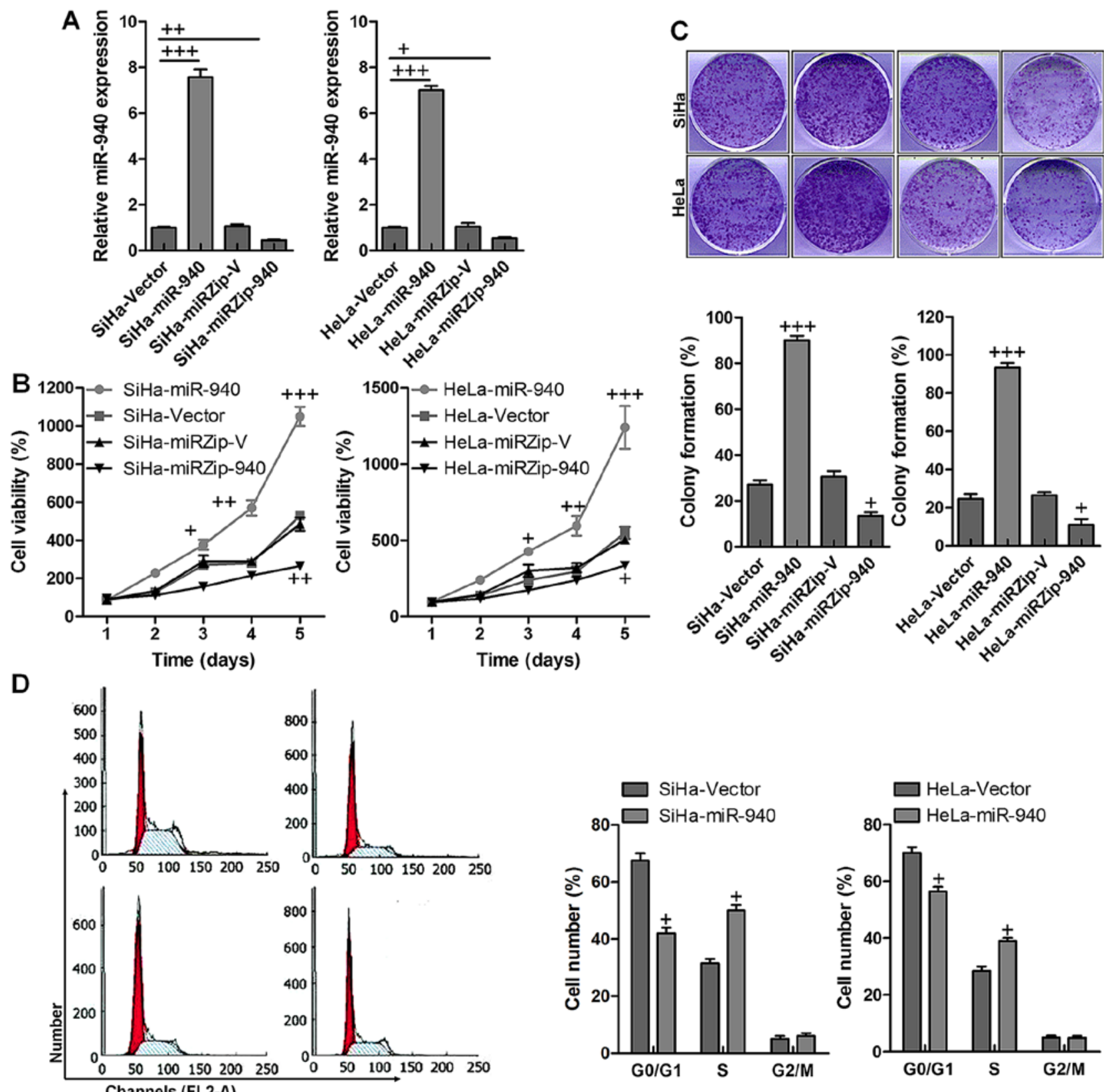

Figure 2. miR-940 stimulated cervical cancer cell proliferation and cell-cycle progression. (A) miR-940 expression levels were calculated by RT-qPCR analysis after transfection under different conditions. (B) Effects of ectopic overexpression of miR-940 or low-expression on the proliferation of the indicated cervical cancer cell lines, as analyzed by the MTT analysis. (C) Representative micrographs (up) and quantification (down) of colonies formed by the indicated cervical cancer cell lines at 14 days after inoculation in colony formation analysis. (D) Flow cytometric analysis of the effect of miR-940 overexpression on cell cycle progression for the indicated cervical cancer cells. Experiments were repeated at least three times with similar results. Data are expressed as the mean \pm SEM. ${ }^{+} \mathrm{P}<0.05,{ }^{++} \mathrm{P}<0.01,{ }^{++} \mathrm{P}<0.001$ versus the normal control.

silenced cells, or the corresponding control cells, were subcutaneously injected into the dorsal flank of nude mice. As shown in Fig. 3C, the tumors formed by miR-940-transduced cervical cancer cells were larger, in both size and weight, than the corresponding control tumors, whereas the tumors formed by miR-940-silenced cervical cancer cells were smaller in size and weight than the corresponding control tumors. These data indicated that miR-940 played an important role in cervical cancer development in vivo.

Expression of miR-940, p27, PTEN and cyclin D1 in human cervical cancer tissues. Finally, to examine whether
miR-940-mediated suppression of p27 and PTEN in cervical cancer is clinically relevant, eight freshly collected cervical cancer samples and two normal cervical tissues were obtained for further study. As shown in Fig. 4, the levels of miR-940 correlated with the protein expression levels of p27, PTEN and cyclin D1. These results suggested that miR-940 decreased the expression of p27 and PTEN and increased cyclin D1 expression, consequently leading to an aggressive phenotype and poorer prognosis in cervical cancer.

p27 and PTEN are essential for miR-940-mediated proliferation in cervical cancer. In an attempt to identify the 


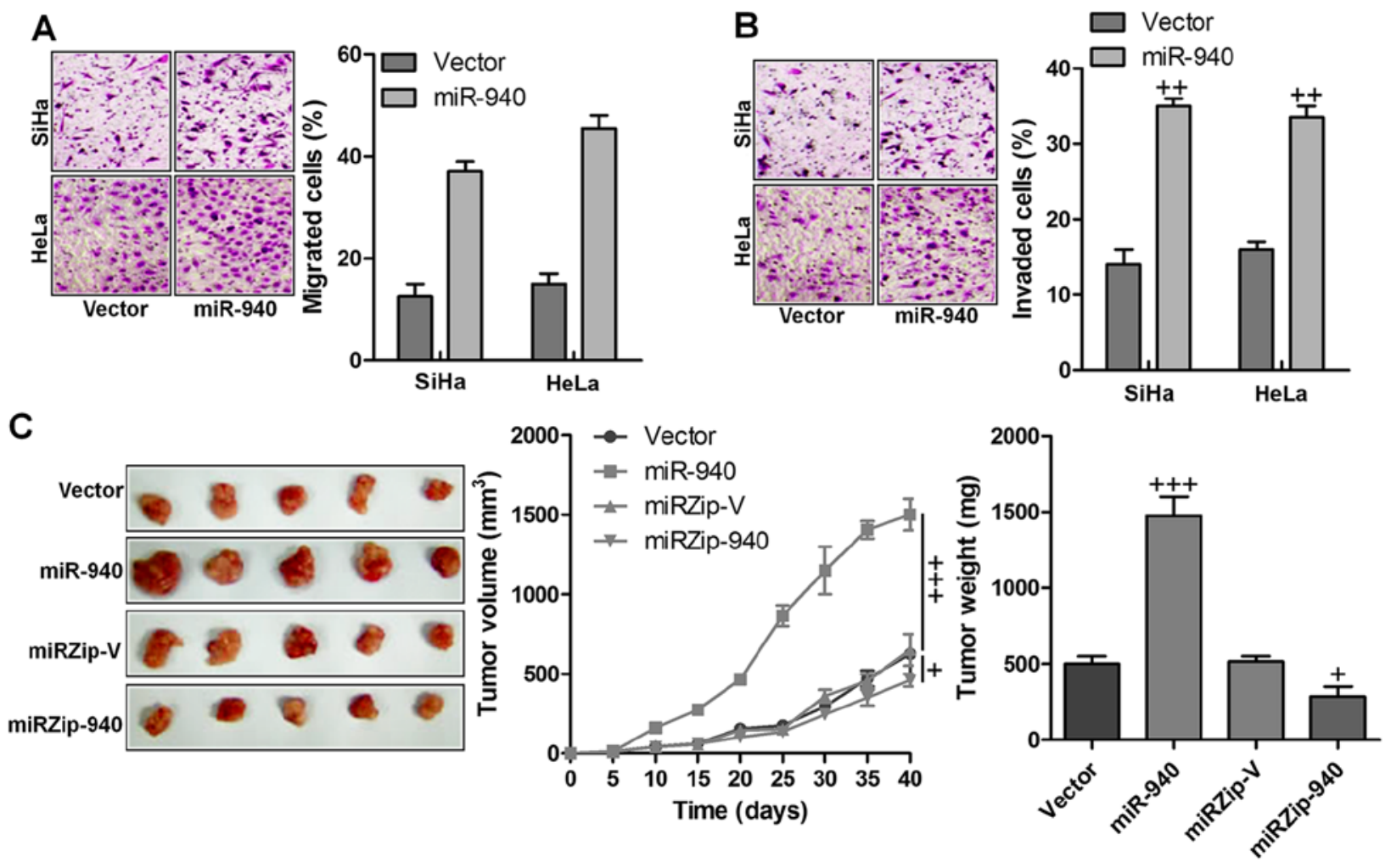

Figure 3. miR-940 suppresses tumorigenicity of cervical cancer cells both in vitro and in vivo. (A) Representative micrographs (left) and quantification (right) of migrated cells in vitro. (B) Representative micrographs (left) and quantification (right) of invaded cells in vitro. (C) Representative images of tumors formed by the indicated cells in the tumor xenograft model. Average tumor volumes $\left(\mathrm{mm}^{3}\right)$ and weight of the tumors for the indicated cells after inoculation. Data are expressed as the mean \pm SEM. ${ }^{+} \mathrm{P}<0.05,{ }^{++} \mathrm{P}<0.01,{ }^{+++} \mathrm{P}<0.001$ versus the normal control.

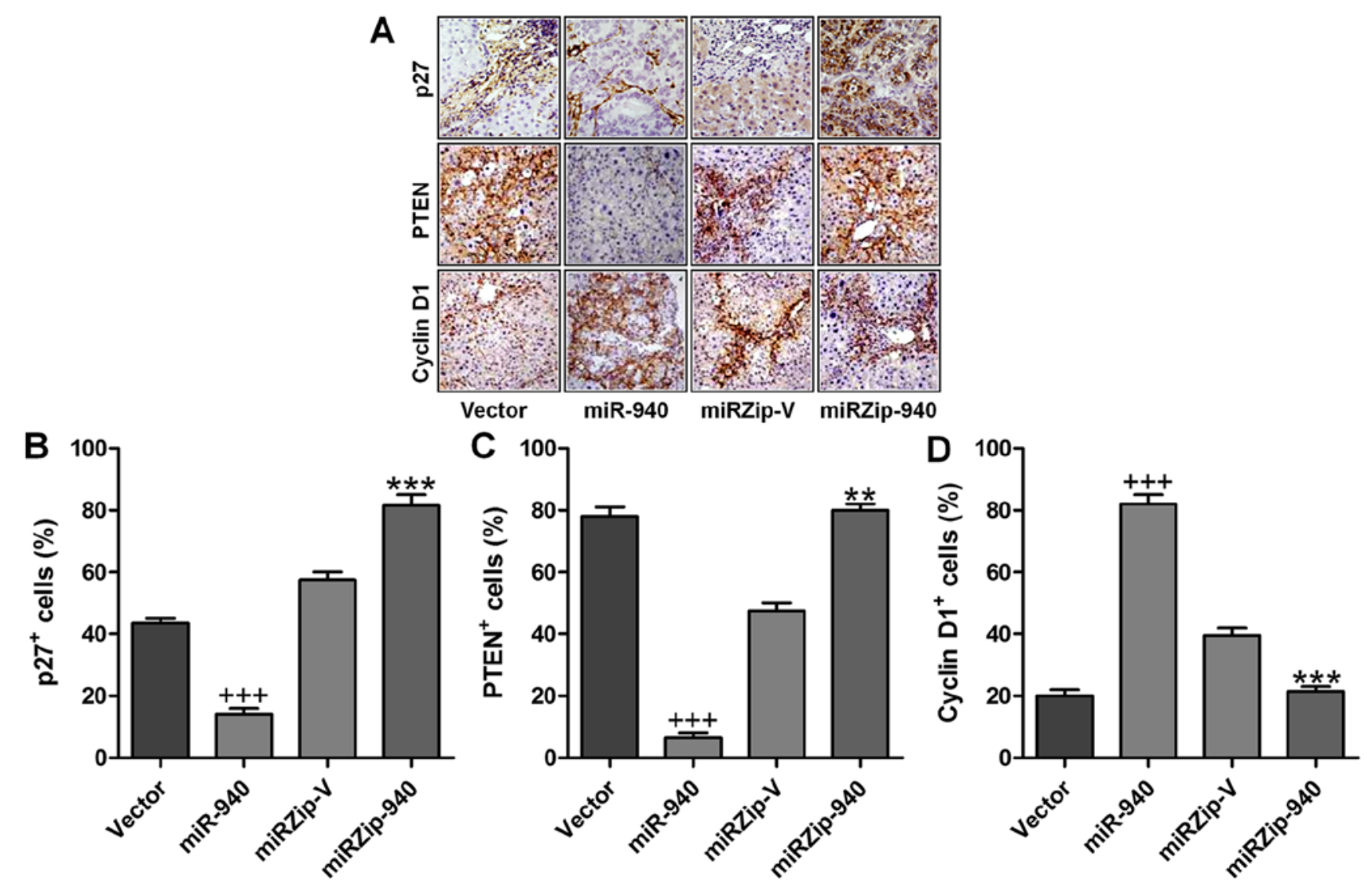

Figure 4. p27 and PTEN are involved in miR-940-mediated cervical cancer progression in vivo. (A) Immunohistochemical analysis of p27, PTEN and cyclin D1 in cervical cancer tumor samples in vivo. Quantification of (B) p27, (C) PTEN and (D) cyclin D1 are shown. Data are expressed as the mean \pm SEM. ${ }^{+} \mathrm{P}<0.05,{ }^{++} \mathrm{P}<0.01,{ }^{+++} \mathrm{P}<0.001$ versus the normal control. 

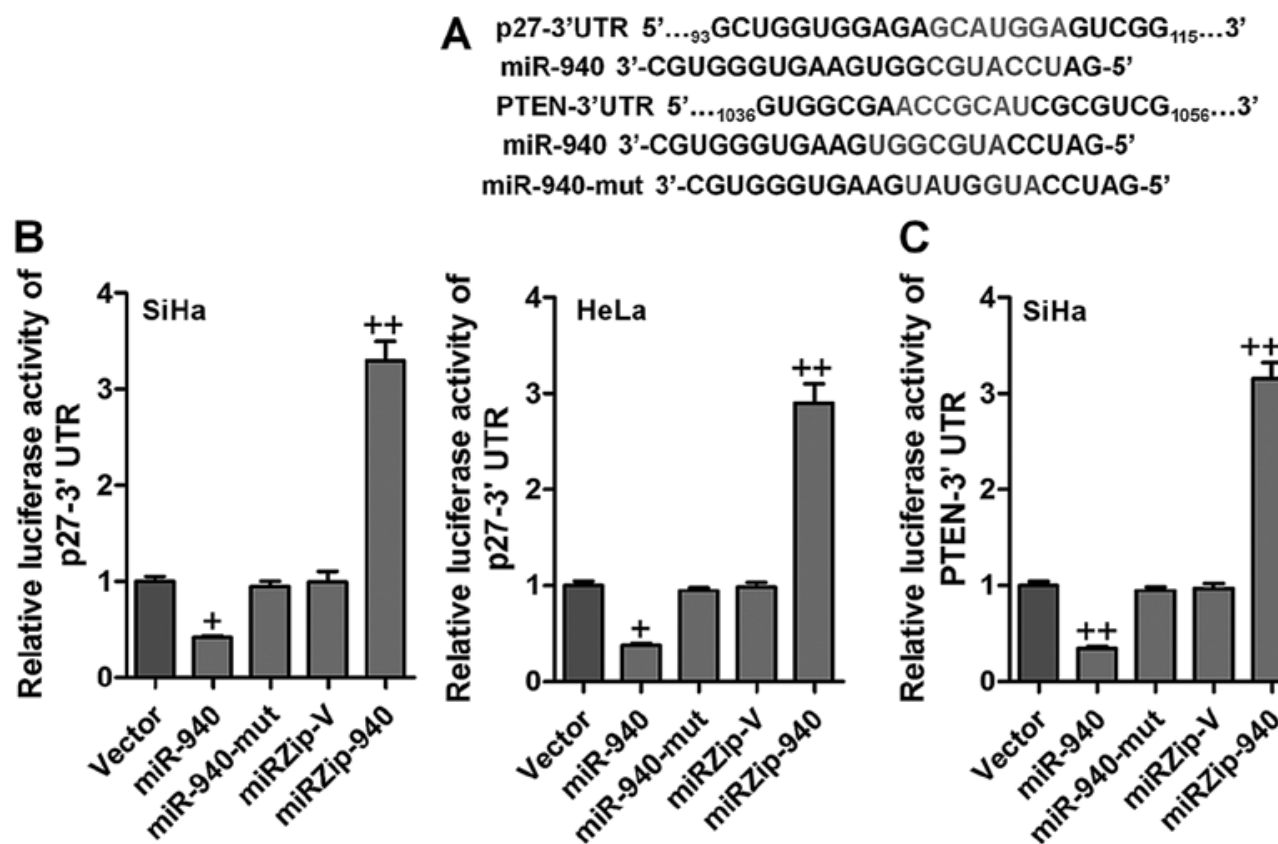

$\mathrm{C}$
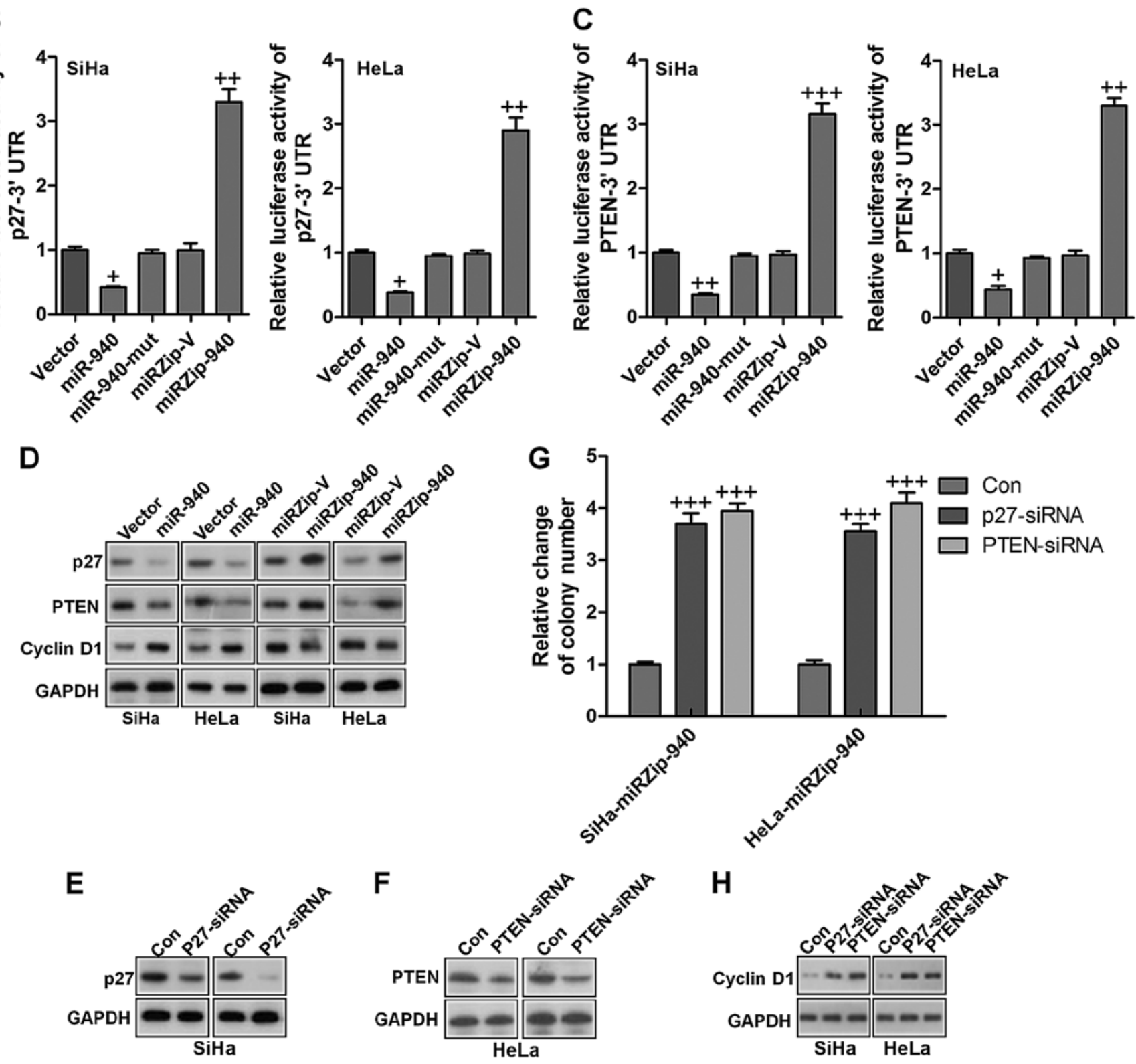

Figure 5. p27 and PTEN are essential for miR-940-mediated proliferation in cervical cancer. (A) Putative target sites for miR-940 in the 3' UTRs of p27 and PTEN and the sequence of miR-940 mutant (miR-940-mut). (B) Luciferase assays of the indicated cells co-transfected with the p27 reporter genes and miR-940, miR-940 inhibitor, miR-940 mutant (miR-940-mut) or the respective controls. (C) Luciferase assays of the indicated cells co-transfected with the PTEN reporter genes and miR-940, miR-940 inhibitor, miR-940 mutant (miR-940-mut) or the respective controls. (D) Western blot analysis of the protein levels of p27, PTEN and cyclin D1 in the indicated cells. Western blot analysis of the protein levels of p27 (E) and PTEN (F) in the indicated cells transfected with specific siRNAs, respectively. (G) Colony formation assay in cervical cancer cells after siRNA treatment of p27 and PTEN. (H) Western blot analysis of cyclin D1 protein expression. Data are expressed as the mean $\pm \mathrm{SEM} .{ }^{+} \mathrm{P}<0.05,{ }^{++} \mathrm{P}<0.01,{ }^{+++} \mathrm{P}<0.001$ versus the normal control.

mRNA targets of miR-940, we performed bioinformatic analysis using a publicly available algorithm (TargetScan 6.2). Additionally, following previous studies, p27 and PTEN have been suggested to be related with miR-940 expression (19-23) Thus, we suppossed that p27 and PTEN might be also potential targets of miR-940 in human cervical cancer. Therefore, they were specificly explored in our study. However, we do not exclude the possibility that there are still other candidates modulated by miR-940. On the contrary, p27 and PTEN are essential for tumor progression. Hence, the two candidates were further explored in our study. As shown in Fig. 5A, p27 and PTEN, which are critical attenuators of cell proliferation and cell-cycle progression, were identified as potential targets of miR-940. Luciferase reporter plasmids containing regions of the 3' UTR of p27 and PTEN were constructed and cotransfected into cervical cancer cells with miR-940, miR-940 

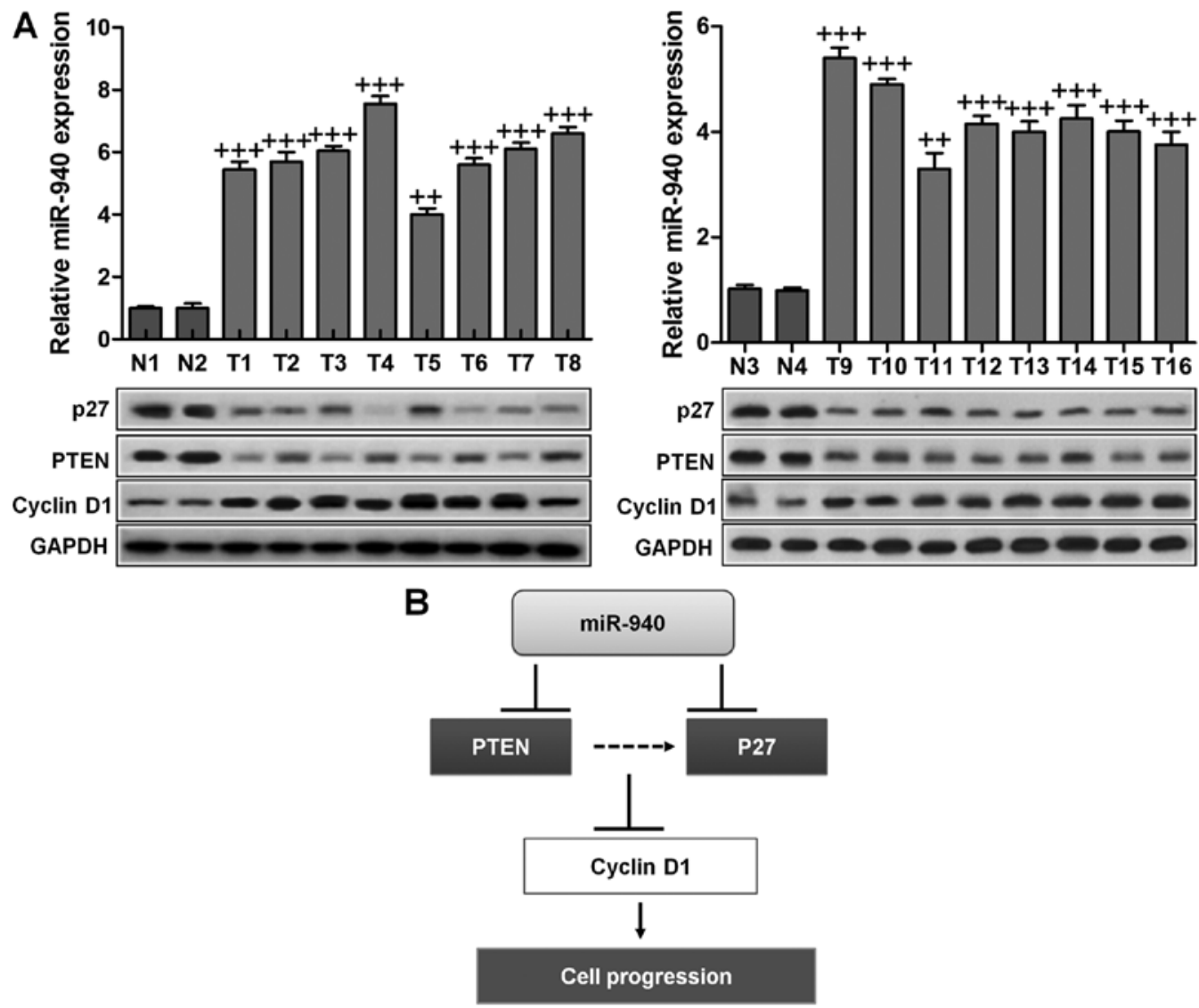

Figure 6. Expression of miR-940, p27, PTEN and cyclin D1 in human cervical cancer tissues. (A) Real-time PCR analysis of miR-940 and western blot analysis of p27, PTEN and cyclin D1 expression in 16 human cervical cancer tissues. (B) Proposed model for the mechanism by which miR-940-mediated downregulation of p27 and PTEN promoted cervical cancer cell proliferation. Data are expressed as the mean $\pm \mathrm{SEM} .{ }^{+} \mathrm{P}<0.05,{ }^{++} \mathrm{P}<0.01,{ }^{++} \mathrm{P}<0.001$ versus the normal control.

inhibitor or the corresponding negative controls. As shown in Fig. 5B and C, miR-940 significantly reduced the luciferase activity of the 27 and PTEN reporter genes, whereas transfection of the miR-940 inhibitor upregulated the luciferase activity of the reporter genes. However, transfection of miR-940-mut (miR-940 mutant) had no significant effect on the luciferase activity of the reporter genes. Western blot analysis showed that ectopic expression of miR-940 markedly decreased, whereas inhibition of miR-940 increased, the protein expression levels of p27 and PTEN in both SiHa and HeLa cervical cancer cells (Fig. 5D). Moreover, the expression of cyclin D1 was increased by ectopic expression of miR-940, and decreased by miR-940 inhibition (Fig. 5D). These results confirmed that p27 and PTEN were direct targets of miR-940. To evaluate the effects of p27 and PTEN on miR-940-induced cervical cancer progression, we suppressed the expression of endogenous p27 and PTEN using specific siRNAs (Fig. 5E and F). The colony formation analysis illustrated that silencing p27 and PTEN increased the proliferation of cervical cells transfected with the miR-940 inhibitor (Fig. 5G). As shown in Fig. 5H, silencing p27 and PTEN the miR-940-inhibitor transfected cells also increased the mRNA and protein expression of cyclin D1, a well-characterized regulator of cell proliferation. These results suggest that silencing p27 and PTEN in miR-940-repressed cells reversed the negative effect of the miR-940 inhibitor on cervical cancer cell proliferation and tumorigenesis.
Expression of miR-940, p27, PTEN and cyclin D1 in human cervical cancer tissues. Finally, to examine whether miR-940-mediated suppression of p27 and PTEN in cervical cancer is clinically relevant, 16 freshly collected cervical cancer samples and four normal cervical tissues were obtained for further study. As shown in Fig. 6A, the levels of miR-940 correlated with the protein expression levels of p27, PTEN and cyclin D1. These results suggested that miR-940 decreased the expression of p27 and PTEN and increased cyclin D1 expression, consequently leading to an aggressive phenotype and poorer prognosis in cervical cancer.

\section{Discussion}

The discovery of effective diagnostic and prognostic biomarkers and therapeutic methods is urgently required for the diagnosis and treatment of cervical cancer. Numerous studies have shown that miRNAs may represent valuable diagnostic and prognostic markers for cancer (24-26). miR-940 is overexpressed in the serum of patients of various carcinoma, and was used to construct a miRNA signature for patients prognosis (16). However, the expression of miR-940 in cervical cancer has not previously been investigated. This study demonstrated that miR-940 is significantly upregulated in cervical cancer. In addition, the expression of miR-940 correlated with cervical cancer progression and overall patient survival, 
indicating that upregulation of miR-940 might contribute to the development of cervical cancer and might have potential as a diagnostic and prognostic biomarker for human cervical cancer.

In this study, p27 and PTEN were both identified as direct targets of miR-940 and could be suppressed by overexpression of miR-940, which in turn increased the proliferation and cell cycle progression of cervical cancer cells in vitro and promoted tumorigenesis in an in vivo model of cervical cancer. Phosphatase and tensin homolog on chromosome 10 (PTEN) is known as a dual-specificity phosphatase, which functions as a tumor suppressor, possessing protein phosphatase and lipid phosphatase activities, disturbing PI3K activation $(27,28)$. In addition, PTEN is functionally related to numerous human cancers. Cells in absence of PTEN have significantly increased levels of PIP3, activating down-streaming signals of PI3K/ AKT targets (29). PTEN overexpression has a close relationship with the activation of proteolytic cascade, contributing to apoptosis, which could be linked to inactivation of AKT (30). Furthermore, PTEN plays an important role in modulating genomic instability as well as DNA repairing signaling pathway $(31,32)$. Recently, a study has indicated that PTEN negatively responds to DNA damage response and has interaction with Chk signaling pathway, leading to cancer progression regulation. Accumulating PTEN could suppress AKT activity, and consequently inhibit cancer cell growth, proliferation and impede apoptosis eventually (27). The effects of p27, a crucial tumor suppressor, are well known including its ability to enhance cancer cell death or suppress cell proliferation permanently. p27 tumor suppressor is a crucial component of a complex network that helps organisms to protect themslves against propagation of cells, which could carry oncogenic mutations (33). Furthermore, p27 is reported to suppress kinase activity and disturb cancer cell cycle development and progression via G1 to S phase (34). Cyclin D1 was overexpressed in a variety of cancers and has a close relationship with different cancer cell proliferation. It mediated cancer cell proliferation through cell cycle progression activation at the point of G1/S restriction (35). p53-induced upregulation of p27 in response to DNA damage induces cell cycle blockade in $\mathrm{G} 1$, followed by DNA repair or induction of apoptosis $(36,37)$.

The evidence discussed above indicates that downregulation of PTEN and p27 may play essential roles in miR-940-induced tumor progression in cervical cancer. However, the detailed regulatory network for miR-940,PTEN and p27, and the related signaling pathways are likely to be complicated and need further investigation. Our study demonstrated that miR-940, which was overexpressed in human cervical cancer, could target and suppress both PTEN and p27, leading to cervical cancer progression. Thus, our results indicated the potential value of miR-940 in cervical cancer development and miR-940 could be considered as useful biomarker for cervical cancer diagnosis and prognosis (Fig. 6B). According to previous studies, target prediction suggested that miR-940 regulated cell signaling, as well as pathways of cell communication and adhesion. These signaling pathways have important effects on cancer initiation and progression, and the downregulation of miR-940 in plasma may perform as a significant biomarker for cancer detection $(17,18)$. These results suggested that the same microRNA can exert distinct biological activities under different cellular contexts.

In conclusion, miR-940 is overexpressed in human cervical cancer and upregulation of miR-940 promoted cervical cancer cell proliferation, cell cycle progression and tumorigenicity both in vitro and in vivo, while inhibition of miR-940 lead to the opposite effects. Furthermore, the function of miR-940 in cervical cancer may be exerted via downregulation of the target genes of PTEN and p27, which play an essential role in the function of miR-940 in cervical cancer. Thus, this study demonstrated that miR-940 might show an important effect on human cervical cancer progression and might represent a potential therapeutic target for cervical cancer therapy.

\section{References}

1. World Health Organization: International Agency for Research on Cancer: Cervical Cancer - Estimated Incidence. Mortality and Prevalence Worldwide in 2012. http://globocan.iarc.fr/old/ FactSheets/cancers/cervix-new.asp. Accession date: 2016-9-10.

2. National Comprehensive Cancer Network: NCCN Clinical Practice Guidelines in Oncology (NCCN Guidelines). Cervical Cancer. Version 1. 2013. https://www.nccn.org/professionals/ physician_gls/f_guidelines.asp. Accession date: 2016-9-10.

3. Monk BJ, Sill MW, McMeekin DS, Cohn DE, Ramondetta LM, Boardman CH, Benda J and Cella D: Phase III trial of four cisplatin-containing doublet combinations in stage IVB, recurrent, or persistent cervical carcinoma: A Gynecologic Oncology Group study. J Clin Oncol 27: 4649-4655, 2009.

4. Thigpen T: The role of chemotherapy in the management of carcinoma of the cervix. Cancer J 9: 425-432, 2003.

5. Kato R, Hasegawa K, Achiwa Y, Okamoto H, Torii Y, Oe S and Udagawa Y: Predicting nedaplatin sensitivity of cervical cancer using the histoculture drug response assay. Eur J Gynaecol Oncol 32: 381-386, 2011.

6. Yamamoto K, Kokawa K, Umesaki N, Nishimura R, Hasegawa K, Konishi I, Saji F, Nishida M, Noguchi H and Takizawa K: Phase I study of combination chemotherapy with irinotecan hydrochloride and nedaplatin for cervical squamous cell carcinoma: Japanese gynecologic oncology group study. Oncol Rep 21: 1005-1009, 2009.

7. Watanabe Y, Nakai H, Etoh T, Kanemura K, Tsuji I, Ishizu A and Hoshiai H: Feasibility study of docetaxel and nedaplatin for recurrent squamous cell carcinoma of the uterine cervix. Anticancer Res 28 (4C): 2385-2388, 2008.

8. Sultana H, Kigawa J, Kanamori Y, Itamochi H, Oishi T, Sato S, Kamazawa S, Ohwada M, Suzuki M and Terakawa N: Chemosensitivity and p53-Bax pathway-mediated apoptosis in patients with uterine cervical cancer. Ann Oncol 14: 214-219, 2003.

9. Zhao Y and Srivastava D: A developmental view of microRNA function. Trends Biochem Sci 32: 189-197, 2007.

10. Bartel DP: MicroRNAs: Genomics, biogenesis, mechanism, and function. Cell 116: 281-297, 2004.

11. Stefani G and Slack FJ: Small non-coding RNAs in animal development. Nat Rev Mol Cell Biol 9: 219-230, 2008.

12. Taipaleenmäki H, Bjerre Hokland L, Chen L, Kauppinen S and Kassem M: Mechanisms in endocrinology: Micro-RNAs: targets for enhancing osteoblast differentiation and bone formation. Eur J Endocrinol 166: 359-371, 2012.

13. Vimalraj S and Selvamurugan N: MicroRNAs: Synthesis, gene regulation and osteoblast differentiation. Curr Issues Mol Biol 15: 7-18, 2013.

14. Ni CW, Qiu H and Jo H: MicroRNA-663 upregulated by oscillatory shear stress plays a role in inflammatory response of endothelial cells. Am J Physiol Heart Circ Physiol 300: H1762-H1769, 2011.

15. Guan YJ, Yang X, Wei L and Chen Q: miR-365: A mechanosensitive microRNA stimulates chondrocyte differentiation through targeting histone deacetylase 4. FASEB J 25: 4457-4466, 2011.

16. Liang D, Xu X, Deng F, Feng J, Zhang H, Liu Y, Zhang Y, Pan L, Liu Y, Zhang D, et al: miRNA-940 reduction contributes to human Tetralogy of Fallot development. J Cell Mol Med 18: 1830-1839, 2014. 
17. Rajendiran S, Parwani AV, Hare RJ, Dasgupta S, Roby RK and Vishwanatha JK: MicroRNA-940 suppresses prostate cancer migration and invasion by regulating MIEN1. Mol Cancer 13: $250,2014$.

18. Song B, Zhang C, Li G, Jin G and Liu C: miR-940 inhibited pancreatic ductal adenocarcinoma growth by targeting MyD88. Cell Physiol Biochem 35: 1167-1177, 2015.

19. Yang HW, Liu GH, Liu YQ, Zhao HC, Yang Z, Zhao CL, Zhang XF and Ye H: Over-expression of microRNA-940 promotes cell proliferation by targeting GSK $3 \beta$ and sFRP1 in human pancreatic carcinoma. Biomed Pharmacother 83: 593-601, 2016.

20. Huang K, Tang Y, He L and Dai Y: MicroRNA-340 inhibits prostate cancer cell proliferation and metastasis by targeting the MDM2-p53 pathway. Oncol Rep 35: 887-895, 2016.

21. Lin SY, Chang $\mathrm{CH}$, Wu HC, Lin CC, Chang KP, Yang CR, Huang CP, Hsu WH, Chang CT and Chen CJ: Proteome profiling of urinary exosomes identifies alpha 1-antitrypsin and $\mathrm{H} 2 \mathrm{~B} 1 \mathrm{~K}$ as diagnostic and prognostic biomarkers for urothelial carcinoma. Sci Rep 6: 34446, 2016.

22. Ma J, Sun F, Li C, Zhang Y, Xiao W, Li Z, Pan Q, Zeng H, Xiao G, Yao K, et al: Depletion of intermediate filament protein Nestin, a target of microRNA-940, suppresses tumorigenesis by inducing spontaneous DNA damage accumulation in human nasopharyngeal carcinoma. Cell Death Dis 5: e1377, 2014.

23. Weber CEM, Luo C, Hotz-Wagenblatt A, Gardyan A, Kordass T, Holland-Letz T, Osen W and Eichmüller SB: miR-339-3p is a tumor suppressor in melanoma. Cancer Res 76: 3562-3571, 2016.

24. Hasegawa $\mathrm{K}$, Kato R, Torii $\mathrm{Y}$, Ichikawa R, Oe S and Udagawa Y: The relationship between ERCC1 expression and clinical outcome in patients with FIGO stage I to stage II uterine cervical adenocarcinoma. Int J Gynecol Cancer 21: 1479-1485, 2011.

25. Chung HH, Kim MK, Kim JW, Park NH, Song YS, Kang SB and Lee HP: XRCC1 R399Q polymorphism is associated with response to platinum-based neoadjuvant chemotherapy in bulky cervical cancer. Gynecol Oncol 103: 1031-1037, 2006.

26. Ozen M, Creighton CJ, Ozdemir M and Ittmann M: Widespread deregulation of microRNA expression in human prostate cancer. Oncogene 27: 1788-1793, 2008.
27. Tamguney T and Stokoe D: New insights into PTEN. J Cell Sci 120: 4071-4079, 2007.

28. Martin J and Dufour JF: Tumor suppressor and hepatocellular carcinoma. World J Gastroenterol 14: 1720-1733, 2008.

29. Yao YJ, Ping XL, Zhang H, Chen FF, Lee PK, Ahsan H, Chen CJ, Lee PH, Peacocke M, Santella RM, et al: PTEN/MMAC1 mutations in hepatocellular carcinomas. Oncogene 18: 3181-3185, 1999.

30. Slipicevic A, Holm R, Nguyen MT, Bøhler PJ, Davidson B and Flørenes VA: Expression of activated Akt and PTEN in malignant melanomas: Relationship with clinical outcome. Am J Clin Pathol 124: 528-536, 2005.

31. Xu Z, Stokoe D, Kane LP and Weiss A: The inducible expression of the tumor suppressor gene PTEN promotes apoptosis and decreases cell size by inhibiting the PI3K/Akt pathway in Jurkat T cells. Cell Growth Differ 13: 285-296, 2002.

32. Weng LP, Brown JL and Eng C: PTEN coordinates G(1) arrest by down-regulating cyclin D1 via its protein phosphatase activity and up-regulating p27 via its lipid phosphatase activity in a breast cancer model. Hum Mol Genet 10: 599-604, 2001.

33. Chu IM, Hengst L and Slingerland JM: The Cdk inhibitor p27 in human cancer: Prognostic potential and relevance to anticancer therapy. Nat Rev Cancer 8: 253-267, 2008.

34. le Sage C, Nagel R and Agami R: Diverse ways to control p27Kip1 function: miRNAs come into play. Cell Cycle 6: 2742-2749, 2007.

35. Chu I, Sun J, Arnaout A, Kahn H, Hanna W, Narod S, Sun P, Tan CK, Hengst L and Slingerland J: p27 phosphorylation by Src regulates inhibition of cyclin E-Cdk2. Cell 128: 281-294, 2007.

36. Vermeulen K, Van Bockstaele DR and Berneman ZN: The cell cycle: A review of regulation, deregulation and therapeutic targets in cancer. Cell Prolif 36: 131-149, 2003.

37. Pateras IS, Apostolopoulou K, Koutsami M, Evangelou K, Tsantoulis P, Liloglou T, Nikolaidis G, Sigala F, Kittas C, Field JK, et al: Downregulation of the KIP family members p27(KIP1) and p57(KIP2) by SKP2 and the role of methylation in p57(KIP2) inactivation in nonsmall cell lung cancer. Int J Cancer 119: 2546-2556, 2006 